Diabetologia 10, 595-600 (1974)

(C) by Springer-Verlag 1974

\title{
Cholesterol Metabolism in the Diabetic Chinese Hamster*
}

\author{
A.V. Chobanian, G.C. Gerritsen, P.I. Brecher, and M. Kessler \\ Cardiovascular Institute, Boston University Medical Center, Boston, Massachusetts, and Diabetes and Atheroselerosis \\ Research, The Upjohn Company, Kalamazoo, Michigan, USA
}

\begin{abstract}
Summary. Cholesterol turnover, pool size, tissue exchange, and tissue content have been examined in normocholesterolemic and hypercholesterolemic diabetic hamsters and matched nondiabetic controls following injection with radiolabelled cholesterol. In the hypercholesterolemic diabetics, turnover time of plasma cholesterol in both rapid and slow exchanging compart. ments was prolonged, and the size of the rapidly exchanging pool was increased. The total production rate of cholesterol did not appear to be altered in the hypercholesterolemic animals. In the normocholesterolemic diabetics, no abnormalities in cholesterol turnover or pool size were apparent. Measurement of tissue specific activities from 2 to 56 days after labelled cholesterol
\end{abstract}

administration suggested that much of the cholesterol in aorta, kidney, adipose tissue, muscle, and brain was in slowly exchanging pools. The rates of exchange of cholesterol between plasma and tissues appeared similar in the diabetic and control groups. Significant increases in tissue cholesterol content were observed in liver, in. testine, aorta, and adipose tissue of the diabetic hypercholesterolemic hamsters. In the normocholesterolemic diabetics, no abnormality in tissue cholesterol content was apparent except for a mild increase in intestinal cholesterol.

Key words: Diabetes mellitus, cholesterol metabolism, hyperlipidemia, tissue cholesterol.
Hyperlipidemia is present in a large percentage of diabetic patients [1] and is an important factor contributing to the high incidence of atherosclerotic disease in such individuals [2]. Minimal information is available concerning the influence of diabetes on cholesterol metabolism. The degree of control of hyperglycemia has been reported to influence serum lipids $[3,4]$ and the majority of patients with elevated level of very low density lipoproteins (VLDL) in plasma exhibit abnormal glucose tolerance [5]. Little association, however, has been noted between the levels of plasma insulin and plasma cholesterol [6].

Hyperlipidemia has also been observed in a number of diabetic animal models. Howard has recently described significant hypertriglyceridemia and prebetalipoproteinemia in monkeys (macaca nigra) with spontaneous diabetes [7]. Experimental studies in alloxandiabetic rats [8] and squirrel monkeys [9] have demonstrated markedly elevated serum cholesterol concentrations when the animals were maintained on a high dietary cholesterol intake. In alloxan-diabetic rats fed a low cholesterol diet, liver cholesterol content has been reported to be significantly increased, even though plasma cholesterol may be normal [10]. Hepatic lipoprotein synthesis and release appeared to be decreased in the rat following alloxan treatment [11] as was the conversion of cholesterol to cholic acid [12]. The fractional turnover of plasma cholesterol has been reported to be reduced and the rapidly exchanging cholesterol compartment increased in alloxan-diabetic squirrel monkeys [9].

We recently have observed spontaneous hypercholesterolemia and hypertriglyceridemia in diabetic

\footnotetext{
* Supported by USPHS Grant 12869 and the U.A.
} Whitaker Fund
Chinese hamsters (cricetulus griseus) $[13]$. In the current investigations we have expanded these studies and have examined the influence of diabetes on tissue sterol content and on cholesterol pool sizes and turnover rates.

\section{Materials and Methods}

The hamsters were mature animals obtained from the Upjohn colony. The diabetic animals had persistent glycosuria and hyperglycemia but no evidence of ketosis. All diabetic animals were matched with a nondiabetic control of the same sex and of similar age and weight. The controls were selected from highly inbred sublines which had not produced diabeties for the past 5 generations. The animals ranged from 6 to 27 months of age and 23 to $39 \mathrm{~g}$ in weight. They were maintained on Purina Mouse Breeder Chow throughout the period of study. The hamsters were injected either intravenously into the jugular vein [14] or intraperitoneally with cholesterol $-7{ }^{3} \mathrm{H}(50 \mathrm{Ci} / \mathrm{mmole})$, which was prepared in $1 \%$ alcoholic saline. The material was administered in a dose of $0.2 \mu \mathrm{Ci} / \mathrm{g}$ body weight and a volume of $0.01 \mathrm{ml} / \mathrm{g}$. Plasma samples were obtained prior to labelled cholesterol administration, at 1, 4, and 7 days following injection, and at weekly intervals thereafter until termination of the study. Blood was obtained from the orbital sinus [15]. Plasma cholesterol was determined by a micromodification of the method of Abell [16], and plasma triglyceride by the densitometric technique of Downing [17]. Plasma cholesterol specific activity was assayed according to our previously described techniques [18]. For tissue specific activity measurements, the animals were sacrificed by decapitation on the second, thirty-fifth, or fifty-sixth day after labelled cholesterol administration. Segments of 
liver, lung, intestine, kidney, epididymal or periovarian fat, quadriceps and pectoralis muscles, skin, brain, and aorta were removed. The tissues were homogenized in chloroform:methanol $(2: 1)$ and the cholesterol content and radioactivity were determined as described [18].

Blood glucose was assayed by the Autoanalyzer microglucose procedure and plasma insulin by a double antibody technique using guinea pig anti-insulin serum [19]. Urinary glucose was estimated by Testape ${ }^{\oplus}$ and urinary ketones by Ketostix ${ }^{\circledR}$. In 5 pairs of animals, lipoprotein typing of plasma was performed by paper electrophoresis [20].

\section{Results}

Both normocholesterolemic and hypercholesterolemic diabetic hamsters were included in the study groups. The normocholesterolemic diabetics and the nondiabetic control animals had fasting plasma cholesterol concentrations averaging below $170 \mathrm{mg} \%$ while the hypercholesterolemic diabetics had plasma ward. No differences in the curves were apparent in the animals injected by either the intraperitoneal or intra. venous route. In one-half of the animals, the curves fitted the model proposed for a 2-pool system [21]. Typical results in a nondiabetic hamster and a hypercholesterolemic diabetic are illustrated in Figures 1 and 2 respectively. In the other half of the animals, the peak level of plasma cholesterol specific activity was not reached in the initial $24 \mathrm{~h}$ after labelled cholesterol administration, and the initial rapidly exchanging compartment could not be estimated because of the limited number of plasma samples obtained during the initial phase of the study. Plasma disappearance curves from the fourth day on were comparable whether or not the specific activity achieved a peak level by $24 \mathrm{~h}$.

The results of the indices of body cholesterol metabolism in the 5 pairs of animals in whom the rapidly exchanging compartment (Pool A) could be measured are summarized in Table 2 . The calculations were made according to the method of Goodman and Noble [21]. The mean $t^{1 / 2}$ of pool $A$ and the mass of pool $A\left(M_{A}\right)$ were somewhat greater in the hypercholesterolemic

Table 1. Blood glucose, insulin, cholesterol and triglycerides in nondiabetic and diabetic hamsters

\begin{tabular}{lrlllc}
\hline & $\mathrm{n}$ & $\begin{array}{l}\text { Blood } \\
\text { Glucose } \\
(\mathrm{mg} \%)\end{array}$ & $\begin{array}{l}\text { Plasma } \\
\text { Insulin } \\
(\mu \mathrm{U} / \mathrm{ml})\end{array}$ & $\begin{array}{l}\text { Plasma } \\
\text { Cholesterol } \\
(\mathrm{mg} \%)\end{array}$ & $\begin{array}{l}\text { Plasma } \\
\text { Triglyceride }\end{array}$ \\
\hline Nondiabetic & 6 & $104 \pm 9$ & $119 \pm 22$ & $131 \pm 11$ & - \\
Normochol. Diabetic & 6 & $281 \pm 20$ & $131 \pm 18$ & $122 \pm 14$ & - \\
Nondiabetic & 15 & $108 \pm \mathbf{3}$ & $121 \pm 11$ & $\mathbf{1 3 6 \pm 9}$ & $195 \pm 42$ \\
Hyperchol. Diabetic & 15 & $298 \pm \mathbf{1 6}$ & $108 \pm 15$ & $247 \pm 13$ & $420 \pm 68$ \\
\hline
\end{tabular}

Values represent the mean $\pm \mathrm{SEM}$.

Glucose, insulin, and triglyceride concentrations were measured in the non-fasting state. Plasma cholesterol and triglyceride concentrations represent the means of at least 4 separate determinations. The triglyceride measurements were performed in only 10 pairs of animals.

cholesterol levels averaging above $200 \mathrm{mg} \%$ (Table 1 ). Plasma cholesterol levels as high as $450 \mathrm{mg} \%$ were observed in the hypercholesterolemic diabetics along: with marked increases in plasma triglycerides. Both diabetic groups had similar elevations of blood glucose concentrations, and neither had significant abnormalities in post-prandial plasma insulin concentrations (Table 1). By electrophoresis, the 2 major plasma lipoprotein groups which appeared increased in the diabetic animals were a fraction with the electrophoretic mobility of a prealbumin and a fraction which did not migrate significantly from the origin. Dr. Charles Day (personal communication) has recently analyzed the plasma lipoproteins of the diabetic animals using ultracentrifugation techniques and has observed increases in the plasma VLDL fraction.

The specific activity time curves for plasma cholesterol during periods of up to 56 days demonstrated a rapid decrease over the first 7 days followed by a slow rate of disappearance which approached an exponential rate of decay from the fourteenth day on- diabetics than in the matched controls. However, the calculated production rate of cholesterol in pool A $\left(\mathrm{PR}_{\mathrm{A}}\right)$ was similar in both groups. The major differences between the hypercholesterolemic diabetics and the nondiabetic controls were in the turnover rates of pool B ( $\mathrm{t} 1 / 2$ pool $\mathrm{B})$ which were significantly increased in the hypercholesterolemic diabetics (Table 3). In the normocholesterolemic diabetics, no change in the turnover rate of pool B was present (Table 3).

Cholesterol content of a variety of tissues was measured in the control and diabetic animals. Tissue cholesterol was significantly increased in the liver, aorta, intestine, and adipose tissue of the hypercholesterolemic animals (Table 4). In the normocholesterolemic diabetics (Table 5), significant increase in tissue cholesterol was present only in the intestine.

Tissue cholesterol specific activities at 35 and 56 days following cholesterol $-7 \cdot{ }^{3} \mathrm{H}$ administration are summarized in Tables 6 and 7 . The changes in tissue specific activities with time in the hypercholesterolemic group are illustrated in Fig. 3. The cholesterol 
specific activities of liver and lung approached that of plasma in each of the time points studied in both diabetic and control animals. The specific activities of aorta, kidney, adipose tissue, and muscle were initially very much lower than those of plasma, but later exceeded the plasma specific activity. The intestinal and skin specific activities showed a modest but consistent decrease below plasma levels while brain specific activity remained very low relative to plasma throughout the study.

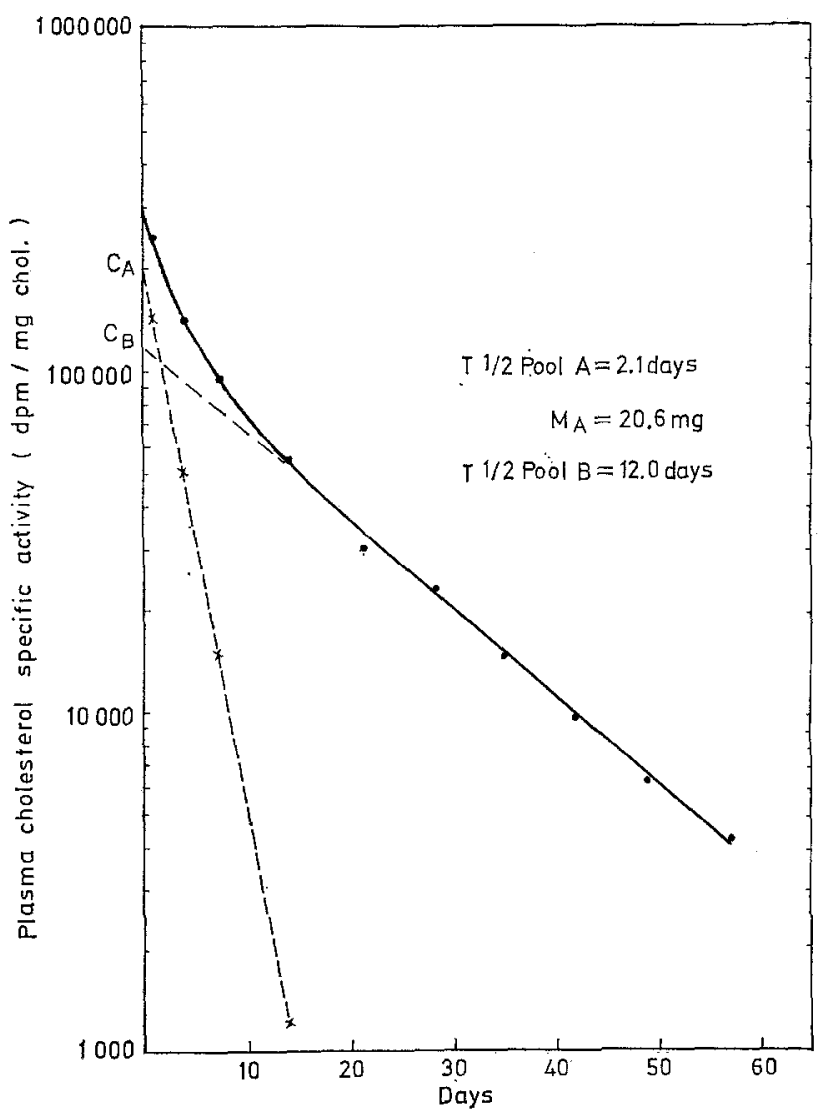

Fig. 1. Disappearance of plasma cholesterol specific activity following the intravenous administration of cholesterol- $7-{ }^{3} \mathrm{H}$ in a representative nondiabetic control hamster

\section{Discussion}

The hypercholesterolemia observed in the diabetic hamster did not appear related directly to the hyperglycemia or insulin abnormalities per se, since no significant differences in blood glucose and plasma insulin concentrations were present between the hypercholesterolemic and normocholesterolemic diabetic groups. Accurate dietary measurements were not made, but since the same diets were fed to both diabetic groups and since the animal weights were comparable, the findings suggest that dietary differences would not be responsible for the observed differences in plasma cho- lesterol. Our preliminary evidence (unpublished observations) suggests that the hypercholesterolemia was more common in certain strains of the diabetic hamster, and it is possible that genetic factors other than those related to the diabetes itself may have influenced the level of serum lipids. However, hypercholesterolemia has never been observed in the nondiabetic Chinese hamsters.

Our studies concerning the lipoprotein patterns in these animals are incomplete, but studies by Day have

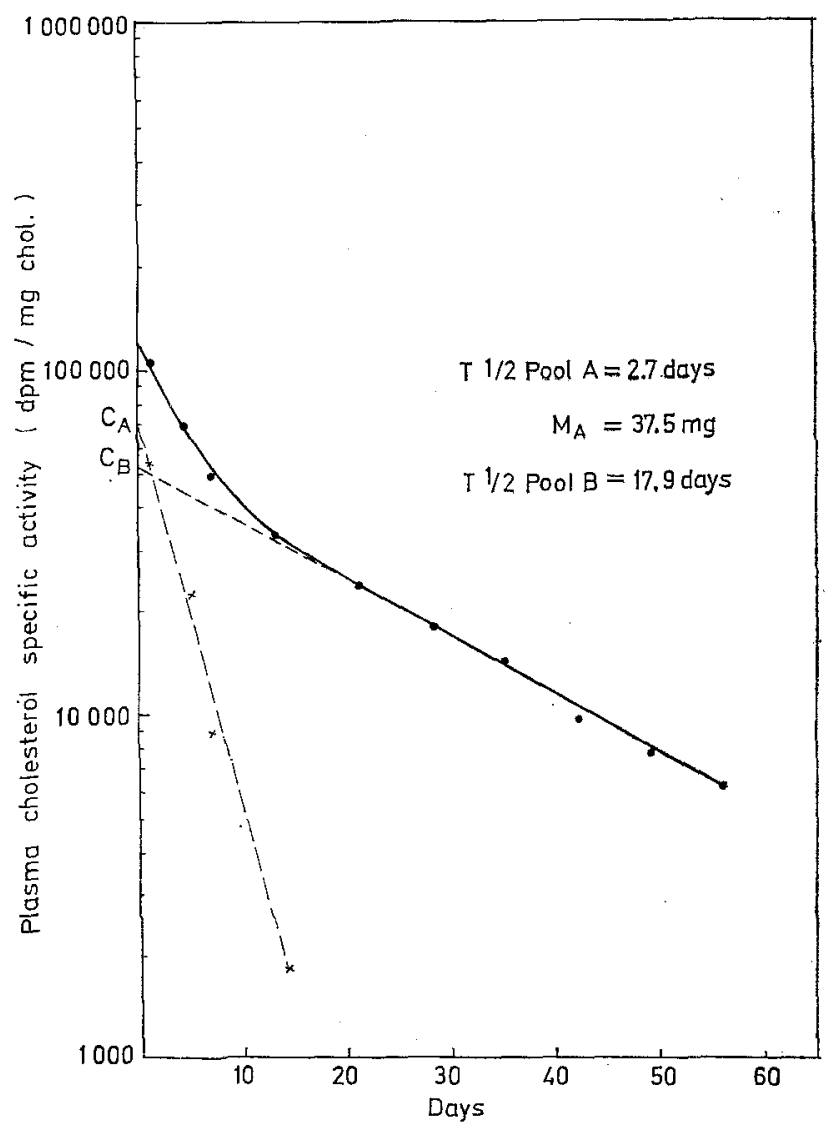

Fig. 2. Disappearance of plasma cholesterol specific activity following the intravenous administration of cholesterol $-7 \cdot{ }^{3} \mathrm{H}$ in a representative diabetic hamster

demonstrated the presence of increased VLDL in the diabetic animals. Recent studies by Bar-On and associates have indicated that apoprotein C-III of VLDL may be increased in streptozotocin-treated rats fed diets high in sucrose [22]. The C-III apoproteins are known inhibitors of lipoprotein lipase, and an increase in their concentration with diabetes conceivably could represent an important factor contributing to the development of the hyperlipidemia.

The fractional turnover of cholesterol in the slowly exchanging compartment (Pool B) was significantly decreased in the hypercholesterolemic diabetics as compared with either the normocholesterolemic diabetics or the control animals. In those animals in which 
pool A measurements could be made, the fractional turnover of cholesterol in pool A was decreased, and the total size of the pool was increased in the hypercholesterolemic diabetics as compared with the controls. Similar results have been observed by Lehner et al. using alloxan-treated squirrel monkeys [9]. The increased size of pool A in the hypercholesterolemic ani-

Table 2. Indices of body cholesterol metabolism in nondiabetic and hypercholesterolemic diabetic hamsters

\begin{tabular}{lcc}
\hline & Nondiabetic & Diabetic \\
\hline Plasma Cholesterol (mg \%) & $148 \pm 25$ & $261 \pm 32$ \\
T $1 / 2$ Pool A (days) & $2.1 \pm 0.2$ & $2.6 \pm 0.3$ \\
$\mathrm{~T} 1 / 2$ Pool B (days) & $12.0 \pm 1.2$ & $15.2 \pm 1.3$ \\
$\mathrm{M}_{\mathrm{A}}$ (mg) & $20.6 \pm 2.4$ & $27.5 \pm 2.9$ \\
$\mathrm{PR}_{\mathrm{A}}$ (mg) & $2.46 \pm 0.31$ & $2.51 \pm 0.36$ \\
\hline
\end{tabular}

Values represent the mean \pm SEM for 5 pairs of animals. $\mathrm{M}_{\mathrm{A}}=$ Mass of pool A.

$\mathrm{PR}_{\mathrm{A}}=$ Production rate of cholesterol in pool A.

Table 3. Fractional turnover rate of cholesterol in the slowly exchanging compartment of nondiabetic and diabetic hamsters

\begin{tabular}{lcll}
\hline Group & n & $\begin{array}{l}\text { Plasma } \\
\text { Cholesterol } \\
\text { (mg\%) }\end{array}$ & $\begin{array}{l}\text { T 1/2 Pool B } \\
\text { (days) }\end{array}$ \\
\hline Nondiabetic & 7 & $\mathbf{1 3 5 \pm 1 2}$ & $11.4 \pm 0.8$ \\
Normochol. Diabetic & 7 & $146 \pm 18$ & $10.7 \pm 0.9$ \\
Nondiabetic & 8 & $\mathbf{1 2 8 \pm 1 5}$ & $11.8 \pm 0.9$ \\
Hyperchol. Diabetic & 8 & $264 \pm 24^{\mathrm{b}}$ & $15.4 \pm 1.4^{\mathrm{a}}$ \\
\hline
\end{tabular}

$$
\text { a } p<0.05
$$

Table 4. Tissue cholesterol content in nondiabetic and hypercholesterolemic diabetic hamsters

\begin{tabular}{lcc}
\hline Tissue & Nondiabetic & $\begin{array}{l}\text { Hypercholesterolemic } \\
\text { Diabetic }\end{array}$ \\
\hline Liver & $11.50 \pm 0.62$ & $15.03 \pm 0.86^{\mathrm{b}}$ \\
Lung & $21.39 \pm 0.87$ & $22.85 \pm 1.34$ \\
Kidney & $17.38 \pm 0.77$ & $17.89 \pm 0.53$ \\
Muscle & $3.59 \pm 0.23$ & $3.72 \pm 0.17$ \\
Intestine & $13.72 \pm 0.92$ & $17.09 \pm 1.35^{\mathrm{a}}$ \\
Fat & $1.18 \pm 0.13$ & $1.57 \pm 0.20^{\mathrm{a}}$ \\
Aorta & $1.53 \pm 0.16$ & $3.18 \pm 0.20^{\mathrm{b}}$ \\
Skin & $9.98 \pm 0.62$ & $9.84 \pm 0.65$ \\
Brain & $88.29 \pm 3.51$ & $85.47 \pm 4.07$ \\
\hline
\end{tabular}

Values represent the mean $\pm S E M$ for 17 pairs of animals. a $p$ value $<0.05$

b $p$ value $<0.01$

mals is not unexpected in view of the marked increase in plasma lipids. The cause of the decreases in fractional turnover of cholesterol is uncertain, although a decrease in conversion of cholesterol to cholic acid has been observed in alloxan-treated rats [12]. In preliminary studies by us, we have been unable to demonstrate any impairment in conversion of cholesterol to 7-d-hydroxycholesterol or bile acids in liver microso- mes from the diabetic hamster. Despite the decreased fractional turnover of cholesterol in the hypercholesterolemic hamsters, the total turnover in pool A was comparable to that of the normocholesterolemic animals. The changes in fractional turnover could reflect some compensatory mechanism for maintaining the total metabolism of cholesterol at relatively constant

Table 5. Tissue cholesterol content in nondiabetic and normocholesterolemic diabetic hamsters

\begin{tabular}{llc}
\hline Tissue & Nondiabetic & $\begin{array}{l}\text { Normocholesterolemic } \\
\text { Diabetic }\end{array}$ \\
& (mg chol/g dry wt) \\
\hline Liver & $10.41 \pm 0.86$ & $12.86 \pm 0.85$ \\
Lung & $20.19 \pm 0.84$ & $19.50 \pm 0.92$ \\
Kidney & $16.50 \pm 0.49$ & $17.64 \pm 1.14$ \\
Muscle & $3.58 \pm 0.46$ & $4.05 \pm 0.26$ \\
Intestine & $13.50 \pm \mathbf{1 . 2 5}$ & $16.62 \pm 1.30^{\mathrm{a}}$ \\
Fat & $1.13 \pm 0.23$ & $0.94 \pm 0.06$ \\
Aorta & $1.61 \pm 0.30$ & $1.70 \pm 0.25$ \\
\hline
\end{tabular}

Values represent the mean \pm SEM for 6 pairs of animals. a $P$ value $<0.05$

Table 6. Relative tissue cholesterol specific activities in nondiabetic and hypercholesterolemic diabetic hamsters 35 days following cholesterol- $7{ }_{-3}^{3} \mathrm{H}$ administration

\begin{tabular}{lll}
\hline Tissue & $\begin{array}{l}\text { Nondiabetic } \\
\text { Tissue chol./plasma chol. }\end{array}$ \\
\hline Plasma & 1.00 & $\begin{array}{c}\text { Diabetic } \\
\text { Liver }\end{array}$ \\
Lung & $0.97 \pm 0.01$ & $0.09 \pm 0.01$ \\
Intestine & $0.99 \pm 0.02$ & $1.00 \pm 0.03$ \\
Kidney & $0.87 \pm 0.05$ & $0.84 \pm 0.05$ \\
Fat & $1.58 \pm 0.05$ & $1.49 \pm 0.11$ \\
Skin & $1.05 \pm 0.11$ & $1.02 \pm 0.13$ \\
Brain & $0.97 \pm 0.07$ & $0.89 \pm 0.07$ \\
Aorta & $0.09 \pm 0.01$ & $0.10 \pm 0.01$ \\
\hline
\end{tabular}

Values represent the mean \pm SEM for 14 pairs of animals. The values are related to plasma cholesterol specific activity (dpm/mg cholesterol) which is arbitrarily designated at 1.00 .

Table 7. Relative tissue cholesterol specific activities in nondiabetic and diabetic hamsters 56 days following cholesterol- \% $^{3} \mathrm{H}$ administration

\begin{tabular}{llll} 
Tissue & $\begin{array}{l}\text { Non- } \\
\text { diabetic }\end{array}$ & $\begin{array}{l}\text { Normo- } \\
\text { cholesterolemic } \\
\text { Diabetic }\end{array}$ & $\begin{array}{l}\text { Hyper- } \\
\text { cholesterolemic } \\
\text { Diabetic }\end{array}$ \\
& \multicolumn{3}{c}{ (Tissue cholesterol/plasma cholesterol) } \\
\hline Plasma & 1.00 & 1.00 & 1.00 \\
Liver & $0.95 \pm 0.03$ & $0.95 \pm 0.05$ & $1.03 \pm 0.05$ \\
Lung & $0.95 \pm 0.03$ & $1.01 \pm 0.04$ & $1.14 \pm 0.17$ \\
Intestine & $\mathbf{0 . 9 0 \pm 0 . 0 6}$ & $0.83 \pm 0.08$ & $0.69 \pm 0.15$ \\
Kidney & $1.56 \pm 0.18$ & $2.37 \pm 0.20$ & $1.79 \pm 0.15$ \\
Fat & $\mathbf{1 . 4 9 \pm 0 . 1 9}$ & $1.75 \pm 0.17$ & $\mathbf{1 . 3 6 \pm 0 . 1 6}$ \\
Muscle & $1.16 \pm 0.14$ & $\mathbf{1 . 7 7 \pm 0 . 4 7}$ & $\mathbf{1 . 2 2 \pm 0 . 0 8}$ \\
\hline
\end{tabular}

Values represent the mean + SEM for 4 non-diabetic animals, 7 normocholesterolemic diabetics, and 4 hypercholesterolemic diabetics. The values are related to plasma cholesterol specific activity (dpm/mg cholesterol) which is arbitrarily designated at 1.00 . 
levels rather than a primary defect in the degradation of removal of cholesterol. The absence of differences in $\mathrm{PR}_{\mathrm{A}}$ between the diabetics and controls suggests that the total amount of new cholesterol entering via dietary intake and endogenous synthesis was comparable in the 2 groups. While such a conclusion depends on the assumption that no significant quantity of cholesterol is excreted from pool $\mathrm{B}$, this assumption appears to be a valid one in view of previous observations in other animals.

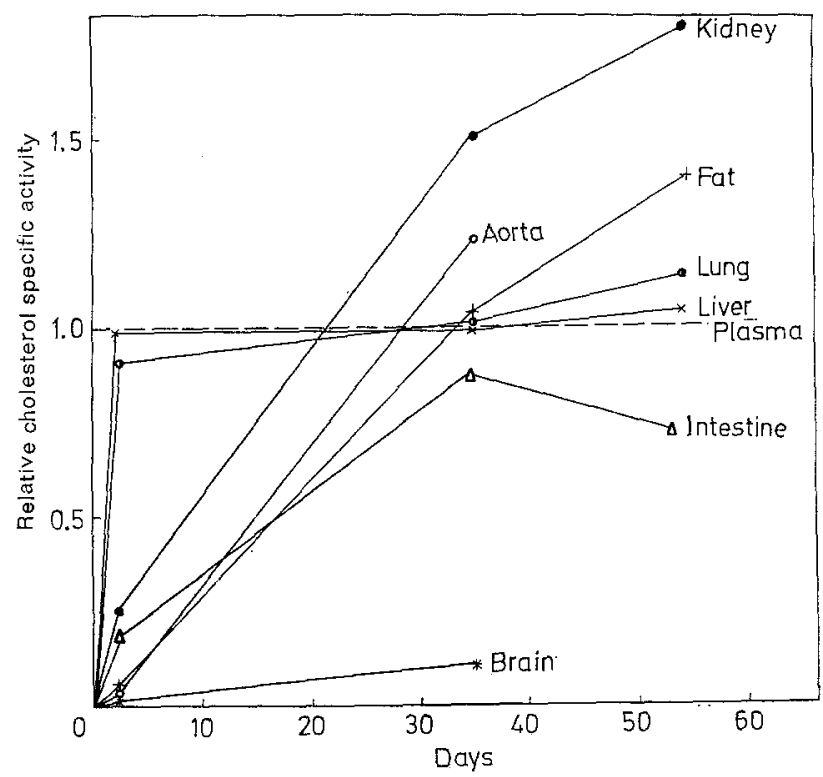

Fig. 3. Tissue cholesterol specific activities at varying time intervals following the intravenous administration of cholesterol- $7 \cdot{ }^{3} \mathrm{H}$ in diabetic hamsters. The ordinate shows the specific activity of cholesterol in different tissues expressed as its ratio to plasma cholesterol specific activity (dpm/mg cholesterol) which is arbitrarily designated at 1.00

The difficulties experienced with determining cholesterol turnover in the rapidly exchanging compartment of some of the animals were related to the fact that the plasma cholesterol specific activity did not always reach a peak by $24 \mathrm{~h}$ after labelled cholesterol administration, and a sufficient number of plasma samples could not be obtained in the first $7-10$ days to permit adequate delineation of the initial phases of the specific activity curve. It is well known that labelled cholesterol, when administered intravenously as a suspension, is taken up rapidly from the circulation, presumably by the reticuloendothelial system [23]. The release back into the circulation is generally complete in $24 \mathrm{~h}$, and thereafter the disappearance curves of cholesterol specific activity are similar, whether the labelled cholesterol is injected initially as a particulate suspension or as lipoprotein-bound material. Our preliminary attempts to complex the labelled cholesterol to hamster lipoproteins have been unsuccessful, and because of the size of the animals and the small total blood volume, we have been unable to bleed the animals more frequently than 4 times per week. However, despite the problems occasionally encountered with determining the turnover of cholesterol in pool $A$, in all instances the fractional turnover rate in the slowly exchanging compartment could be assessed.

The tissue specific activities of kidney, adipose tissue, muscle, and aorta initially were much lower than that of plasma but exceeded the plasma specific activity at 5 and 8 weeks after administration of label. The results suggest that much of the cholesterol in these tissues is in a very slow exchanging pool. The relatively low specific activity in intestine throughout the study period is somewhat surprising. It could reflect a large pool of unlabelled sterol which has been absorbed from the diet and has not come into equilibrium with plasma cholesterol. Significant endogenous synthesis of cholesterol also could contribute to the low specific activities observed in the intestine.

Our calculations have assumed the presence of a 2-pool system. It is possible that the very slowly exchanging pools of cholesterol recently described in human subjects [24] may also be present in the hamster. Accurate estimations of the size of pool B would require knowledge concerning the existence of such additional pools.

The increase in arterial cholesterol content in the hypercholesterolemic diabetic animals is consistent with our previous findings [13]. Aortic lesions have been observed by us in these animals [25] and may reflect the presence of early atherosclerotic disease. No increased aortic lipid synthesis has been observed in the diabetic hamster [13], and a major determinant of the increased arterial lipid content may be the level of serum lipids and lipoproteins [13].

The increase in cholesterol content of adipose tissue in the hypercholesterolemic hamsters is of interest although the cause of the increase is uncertain. The tissue specific activity measurements suggested that the adipose tissue cholesterol was primarily in a very slowly exchanging pool. Previous studies have indicated that adipose tissue is an important storage site of cholesterol [26]. In man, the size of pool B and the $\mathrm{PR}_{\mathrm{A}}$ appear to be related significantly to the degree of adiposity [27]. The hamsters which we studied did not appear to be obese, and no abnormality in cholesterol pool size or turnover would be expected on the basis of increased fat content.

Acknowledgment. The authors wish to express their thanks to Margaret Blanks for her expert technical assistance.

\section{References}

1. Barach, J.H., Lowry, A.D.: Lipoprotein molecules, cholesterol, and atherosclerosis in diabetes mellitus. Diabetes 1, $441-446(1952)$

2. Entmacher, P.S., Marks, H.H.: Diabetes in 1964. A world survey. Diabetes 14, 212-223 (1965) 
3. Bierman, E.L., Dole, V.P., Roberts, T.N.: An abnormality of non-esterified fatty acid metabolism in diabetes mellitus: Diabetes 6, $475-479$ (1957)

4. Adlersberg, D., Eister, L.: Circulatory lipids in diabetes mellitus. J. Amer. med. Ass. 170, 1261 - 1265 (1959)

5. Fredrickson, D.S., Levy, R.I., Lees, R.S.: Fat transport in lipoproteins - an integrated approach to mechanism and disorders. New Engl. J. Med. 276, 273-281 (1967)

6. Abrams, M.E., Jarrett, R.J., Keen, H., Boyns, D.R., Crossley, J.N.: Oral glucose tolerance and related factors in a normal population sample. II. Interrelationship of giycerides, cholesterol and other factors with the glucose and insulin response. Brit. med. J. 1969 I, $599-602$

7. Howard, C.F., Jr.: Correlations of serum triglyceride and prebetalipoprotein levels to the severity of spontaneous diabetes in macaca nigra. J. clin. Endocr. 38, $856-860(1974)$

8. Winegrad, A.I., Yalcin, S., Mulcahy, P.D.: Alterations in aortic metabolism in diabetes. In: On the Nature and Treatment of Diabetes, (Leibel, B.S., Wrenshall, G.A., eds.) pp. 452-462. Amsterdam: Excerpta Medica Foundation 1965

9. Lehner, N.D.M., Clarkson, T.B., Bell, F.P., St. Clair, R.W., Lofland, H.B.: Effects of insulin deficiency, hypothyroidism, and hypertension on cholesterol metabolism in the squirrel monkey. Exp. molec. Path. 16, 109-123 (1973)

10. Sadahiro, R., Takeuchi, N., Yamamura, V.: Cholesterol metabolism in the alloxan-diabetic rat. Proc. Symp. chem. Physiol. Path. 7, 177 (1967)

11. Wilcox, H.G., Dishmon, G., Heimberg, M.: Hepatic lipid metabolism in experimental diabetes. IV. Incorporation of amino acid ${ }^{14} \mathrm{C}$ into lipoprotein-protein and triglyceride. J. biol. Chem. 243, 666-675 (1968)

12. Sadahiro, R., Takeuchi, N., Kumagai, A., Yamamura, Y.: Studies on cholesterol metabolism in experimental diabetic rat. Endocr. jap. 17, 225-232 (1970)

13. Chobanian, A.V., Gerritsen, G.C., McCombs, L., Brecher, P.I.: Arterial lipid metabolism in diabetic animal models with reduced or elevated plasma insulin levels. In: Proceedings of the Third International Symposium on Atherosclerosis. Berlin, Heidelberg, New York: Springer 1974 (in press)

14. Kassel, R., Levtan, S.: A jugular technique for the repeated bleeding of small animals. Science $\mathbf{1 1 8}$, $563-564(1953)$

15. Riley, V.: Adaptation of orbital bleeding technique to rapid serial blood studies. Proc. Soc. exp. Biol. (N.Y.) 104, 751-754 (1960)

16. Abell, L. L., Levy, B. B., Brodie, B.B., Kendall, F.E.: A simplified method for the estimation of total cholesterol in serum and demonstration of its specificity. J. clin. Chem. 195, 357 (1952)

17. Downing, D.T.: Photodensitometry in the thin-layer chromatographic analysis of neutral lipids. J. Chromatog. 38, 91 -99 (1968)

18. Chobanian, A.V., Hollander, W.: Body cholesterol metabolism in man. $I$. The equilibration of serum and tissue cholesterol. J. clin. Invest. 41, 1732-1737 (1962)

19. Gerritsen, G.C., Dulin, W.E.: Characterization of diabetes in the Chinese hamster. Diabetologia 3, 74 (1967)

20. Lees, R.S., Hatch, F.T.: Sharper separation of lipoprotein species by paper electrophoresis in albumincontaining buffer. J. Lab. clin. Med. 61, 518-528 (1963)

21. Goodman, D.S., Noble, R.P.: Turnover of plasma cholesterol in man. J. clin. Invest. 47, 231-241 (1968)

22. Bar-On, H., Roheim, P.S., Eder, H.A.: Apoprotein composition and lipoprotein lipase (LPL) activation in experimental diabetes mellitus. J. clin. Invest. 53, 4 a (1974)

23. Nilsson, A., Zilversmit, D.B.: Fate of intravenously administered particulate and lipoprotein cholesterol in the rat. J. Lipid Res. 13, 32-38 (1972)

24. Samuel, P., Perl, W., Holtzman, C.M., Rochman, N.D., Lieberman, S.: Long-term kinetics of serum and xanthoma cholesterol radioactivity in patients with hypercholesterolemia. J. clin. Invest. 51, 266-278 (1972)

25. MeCombs, H.L., Gerritsen, G.C., Chobanian, A.V.: Morphological changes in the aorta in the diabetic Chinese hamster. Diabetologia 10, 601-606 (1974)

26. Angel, A., Farkas, J.: Structural compartments in adipose cells. In: Adipose Tissue: Regulation and Metabolic Function. pp. 152-161. London: Academic Press 1970

27. Nestel, P.J., Whyte, H.M., Goodman, D. S.: Distribution and turnover of cholesterol in humans. J. clin. Invest. 48, 982-991 (1969)

Dr. Aram V. Chobanian

Cardiovascular Institute

Boston University Medical Center

Boston, Mass. 02118

USA 